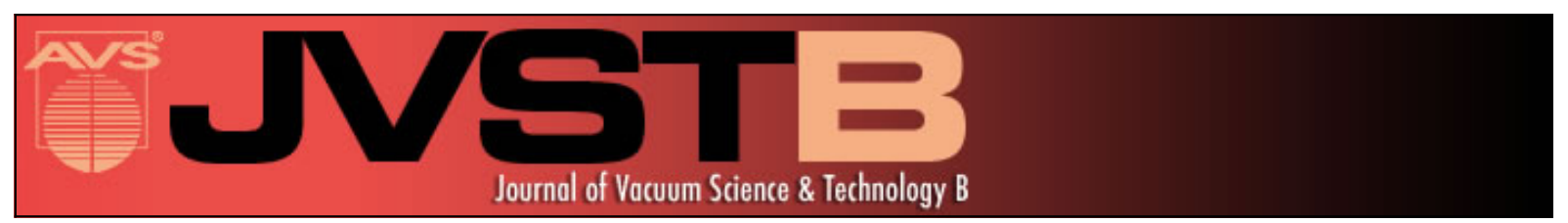

\title{
Concept for assembling individual nanostructure-based components into complex
} devices

Dmitry B. Suyatin, Richard Sundberg, Ivan Maximov, Sergey Shleev, and Lars Montelius

Citation: Journal of Vacuum Science \& Technology B 33, 062202 (2015); doi: 10.1116/1.4931952

View online: http://dx.doi.org/10.1116/1.4931952

View Table of Contents: http://scitation.aip.org/content/avs/journal/jvstb/33/6?ver=pdfcov

Published by the AVS: Science \& Technology of Materials, Interfaces, and Processing

\section{Articles you may be interested in}

Rectifying characteristics of Te-doped GaAs nanowires

Appl. Phys. Lett. 99, 182102 (2011); 10.1063/1.3658633

Gelatin/glycerol coating to preserve mechanically compliant nanowire electrodes from damage during brain implantation

J. Vac. Sci. Technol. B 28, C6K13 (2010); 10.1116/1.3498764

High-performance GaN-based light-emitting diode using high-transparency $\mathrm{Ni} / \mathrm{Au} / \mathrm{Al}$-doped $\mathrm{ZnO}$ composite contacts

Appl. Phys. Lett. 87, 181107 (2005); 10.1063/1.2120913

Presence of nanosize Au dots on the formation of ohmic contact for the Ni-Au base film to $p$-GaN

J. Vac. Sci. Technol. B 23, 2127 (2005); 10.1116/1.2041651

Pd versus Au as evaporated metal contacts to molecules

Appl. Phys. Lett. 86, 042113 (2005); 10.1063/1.1854742

\section{HDDEN}

\section{Instruments for Advanced Science}

w www.HidenAnalytical.com E info@hiden.co.uk

CLICK TO VIEW our product catalogue

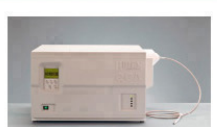

Gas Analysis

dynamic measurement of reaction gas steans

catalysis and thermal analysis

molecular beam studies

dissolved species probes

fermentation environmental and ecological studies

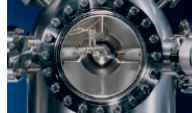

Surface Science

UHVTPD

SIMS demental imaging - surface mapping

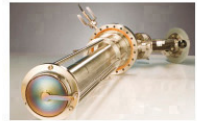

Plasma Diagnostics plasma source characterization etch and deposition process reaction

analysis of neutral and radical species

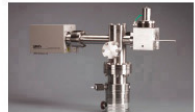

Vacuum Analysis , partial pressure measurement and contro of process gases reactive sputter process contro vacuum diagnostics Taum coatin process monitorin 


\title{
Concept for assembling individual nanostructure-based components into complex devices
}

\author{
Dmitry B. Suyatin \\ Division of Solid State Physics, Neuronano Research Center, and The Nanometer Structure Consortium \\ (nmC@LU),Lund University,Box118,Lund SE-22100,Sweden \\ Richard Sundberg \\ Division of Solid State Physics, and The Nanometer Structure Consortium (nmC@LU), Lund University, \\ Box 118, Lund SE-22100, Sweden and Department of Biomedical Sciences, Faculty of Health and Society, \\ Malmö University, Jan Waldenströms Gata 25, Malmö SE-21428, Sweden \\ Ivan Maximov \\ Division of Solid State Physics, and The Nanometer Structure Consortium (nmC@LU), Lund University, \\ Box 118, Lund SE-22100, Sweden \\ Sergey Shleev \\ Department of Biomedical Sciences, Faculty of Health and Society, Malmö University, Jan Waldenströms \\ Gata 25, Malmö SE-21428, Sweden and Laboratory of Chemical Enzymology, A.N. Bach Institute of \\ Biochemistry, Russian Academy of Sciences, Leninsky Prospect 33, Moscow RU-119071, Russia \\ Lars Montelius ${ }^{\text {a) }}$ \\ Division of Solid State Physics, Neuronano Research Center, and The Nanometer Structure Consortium \\ (nmC@LU),Lund University,Box118,Lund SE-22100,Sweden
}

(Received 11 July 2015; accepted 17 September 2015; published 15 October 2015)

\begin{abstract}
Minute electronic (bio)devices will likely play an increasingly important role in everyday life and beyond, as overall device size often limits device functionality and applicability, a factor especially critical for brain implants. Recent progress in micro- and nanoelectronics has enabled the production of nanoscale electronic components; however, overall device size is often defined by technical and technological limitations, in particular, the ability to combine heterogeneous components made using incompatible processes on different substrates. Here, the authors suggest and evaluate a concept and approach aimed at the direct three-dimensional assembly of individual nanoscale-based components into complex devices for brain implants. They demonstrate this assembly possibility via the transfer of free-standing GaP nanowires, as well as test devices made of gold film which exhibit good quality electrical contacts. The key features essential for such a functional assembly process are discussed. The authors expect this approach to be generic and to enable the development of complex minute electronic (bio)devices based on nanoscale components. The proposed type of assembly may be especially beneficial for devices with strict size constraints, such as implantable neural interfaces. (C) 2015 American Vacuum Society.
\end{abstract}

[http://dx.doi.org/10.1116/1.4931952]

\section{INTRODUCTION}

The miniaturization of electronic devices is a long-lasting trend, the effects of which can be seen widely in everyday life. Indeed, the realization of minute electronic devices composed of nanoscale electronic components may bring this miniaturization trend to a new level, ${ }^{1}$ providing new opportunities in different areas such as biomedicine, ${ }^{2-6}$ environmental sensing, ${ }^{7}$ electronics ${ }^{8}$ and aerospace applications, ${ }^{9,10}$ and potentially enabling the development of devices and structures currently unattainable with present technologies. Extreme size downscaling is especially critical for implantable biomedical devices ${ }^{6,11}$ such as brain implants, ${ }^{12,13}$ for which an inappropriate size may lead to a complete degradation of device functionality due to biological tissue damage,

\footnotetext{
a) Present address: International Iberian Nanotechnology Laboratory, Av. Mestre Jose Varga, 4715-330 Braga, Portugal; electronic mail: Lars.Montelius@ftf.lth.se
}

with nanostructures potentially providing a number of important advantages. ${ }^{14}$

Recent progress in nanotechnology has enabled the fabrication of a range of very complicated and highly efficient nanoscale devices. For example, all the individual components required for the construction of self-contained neuronal probes powered by biofuel cells (Fig. 1) have already been developed, and some have even been tested on living organisms (in vivo). ${ }^{15-18}$ However, the integration of heterogeneous (dissimilar) nanoscale components, which is required for self-contained probes, remains a challenge. So far such integration has been demonstrated only for micrometer-scale components, ${ }^{12,19-25}$ and it has been shown that heterogeneous integration can be done in a very efficient way by parallel and unsupervised self-assembly. ${ }^{26-29}$

Micro- and nanoscale components are typically fabricated on rather thick (down to $25 \mu \mathrm{m}$, typically $200-300 \mu \mathrm{m}$ thick) and large (down to $25 \mu \mathrm{m}$, typically $5-15 \mathrm{~mm}$ lateral dimensions) dies due to practical limitations regarding die 


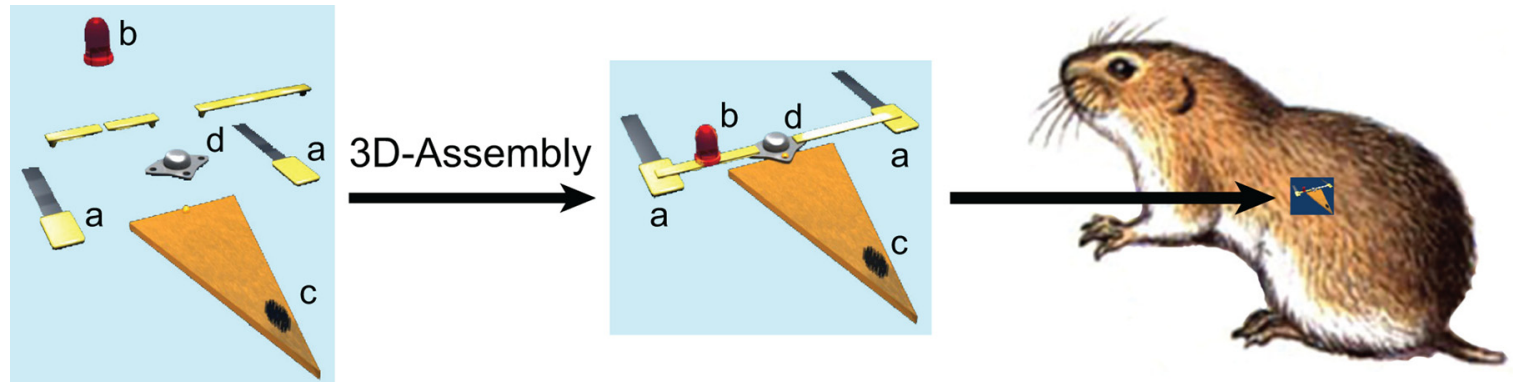

FIG. 1. (Color online) Concept and potential of the direct three-dimensional assembly of individual nanostructure-based components into complex devices: enzyme-modified nanoelectrodes of a biofuel cell (a), nanowire-based light emitting diode (b), nanowire-based nano(bio)sensor (c), and an operational unit (d), fabricated on different substrates, may be assembled together. After biomodification this may lead to a functional complex self-contained nanobiodevice for minimally invasive neuronal interfacing, a device not currently attainable with present technologies.

handling. ${ }^{26,30}$ It is currently unclear as to how such devices can be (1) isolated from the bulk substrate on which they are fabricated, (2) combined with heterogeneous (incompatible from a technological process point of view) micro- and nanoscale structures/functional elements, and (3) assembled in three dimensions (3D). These problems limit overall device size and the advantages which nanostructures can offer, in particular, to brain implants. Thus, the main objective of the present work was to study the controlled 3D assembly of individual heterogeneous nanostructure-based components.

Here, we suggest and test a concept and approach aimed at the direct 3D assembly of individual heterogeneous nanostructure-based components into complex devices dedicated for brain implants. A schematic of this concept for the fabrication of complex (bio)devices is presented in Fig. 1. We demonstrate that one way to achieve both isolation from the original substrate and the 3D integration of heterogeneous micro- and nanoscale structures is to design and form special blocks enabling the optimal protection, handling and performance of each component. We prove the feasibility of this type of assembly by transferring blocks containing freestanding GaP nanowires (NWs) grown by epitaxy on high quality crystalline substrate to another substrate, using a dual, electron and ion, beam system [focused gallium ion beam (FIB) and scanning electron microscope (SEM)] equipped with a gas injection system (GIS) and a micromanipulator. We do this by encapsulating the NWs in a block suitable for damage-free NW transfer. We also evaluate contact resistance for test devices made of gold $(\mathrm{Au})$ film and show that the contact resistance to components assembled via the proposed approach is suitable for device applications.

\section{EXPERIMENT}

Our work was driven by the practical implementation of brain implants based on free-standing NWs for in vivo applications. This determined the design of our experiments, for which we chose free-standing GaP NWs of the same type as were previously used in neural interfaces. ${ }^{17,31,32}$

The suggested approach, which we describe herein, includes the following steps: (1) the formation of special blocks encapsulating individual nanostructure-based components [Figs. 2(a)-2(c)], (2) block detachment and manipulation [Fig. 2(d)], (3) attachment of the blocks at a new locus
[Fig. 2(e)], and (4) component activation by, e.g., removing the component protection [Fig. 2(f)].

The experiments were performed using an FIB-SEM (xT Nova NanoLab 600, FEI Company) equipped with a FIB system, SEM, a manipulator (OmniProbe 100.7, Oxford Instruments) with a tungsten (W)-needle probe, and a GIS for beam assisted deposition of W. This tool was used to deposit protection structures, cut out, separate, transfer, and fix blocks with free-standing NWs and the test devices. Beamassisted deposition of $\mathrm{W}$ was carried out via FIB-induced chemical vapor deposition (W-FIB-CVD) from $\mathrm{W}(\mathrm{CO})_{6}$ precursor gas. ${ }^{33}$

\section{A. Transfer of free-standing nanowires}

Vertical free-standing GaP NWs were epitaxially grown on the (111)B GaP surface of $300 \mu \mathrm{m}$ thick GaP substrates using metalorganic vapor phase epitaxy (MOVPE, AIX200/ 4, Aixtron AG) from Au aerosol catalytic particles. ${ }^{34,35}$

Attempts at the direct transfer of free-standing NWs lead to the destruction of the NWs by the ion beam and the material redeposition during FIB milling. To overcome this problem, we chose to construct special blocks with which to encapsulate and protect the NWs, thereby enabling their direct manipulation and transfer.

For the formation of these special blocks, we used a $\sim 1 \mu \mathrm{m}$ thick shield cut from Au film via FIB milling [Fig. 2(a)]. The Au film itself was prepared by the thermal evaporation of $\mathrm{Au}$ (custom-built thermal evaporator, base pressure $<10^{-6}$ mbar) on a substrate covered with polymethylmethacrylate (PMMA A5 $950 \mathrm{k}$, spun at $5000 \mathrm{rpm}$ for $30 \mathrm{~s}$ and subsequently baked for $15 \mathrm{~min}$ on a hot plate at $160^{\circ} \mathrm{C}$ ) and then treated in acetone $\left(60^{\circ} \mathrm{C}\right)$ for $30 \mathrm{~min}$, rinsed in isopropanol and blow dried with nitrogen stream. This process produced a $1 \mu \mathrm{m}$ thick Au film which sat loosely on the substrate, thereby facilitating the lifting of the FIB-cut Au film pieces.

The shield was also designed to include a special bridge for micromanipulator attachment. After the W-needle of the manipulator was soldered to this bridge via W-FIB-CVD, the shield was moved on top of the NWs [Fig. 2(a)], attached to the substrate and the NWs were sealed by W-FIB-CVD. The bridge, to which the manipulator was connected, was then cut free [Fig. 2(b)]. Following this a block with sealed NWs was cut from the substrate [Fig. 2(c)], forming a 

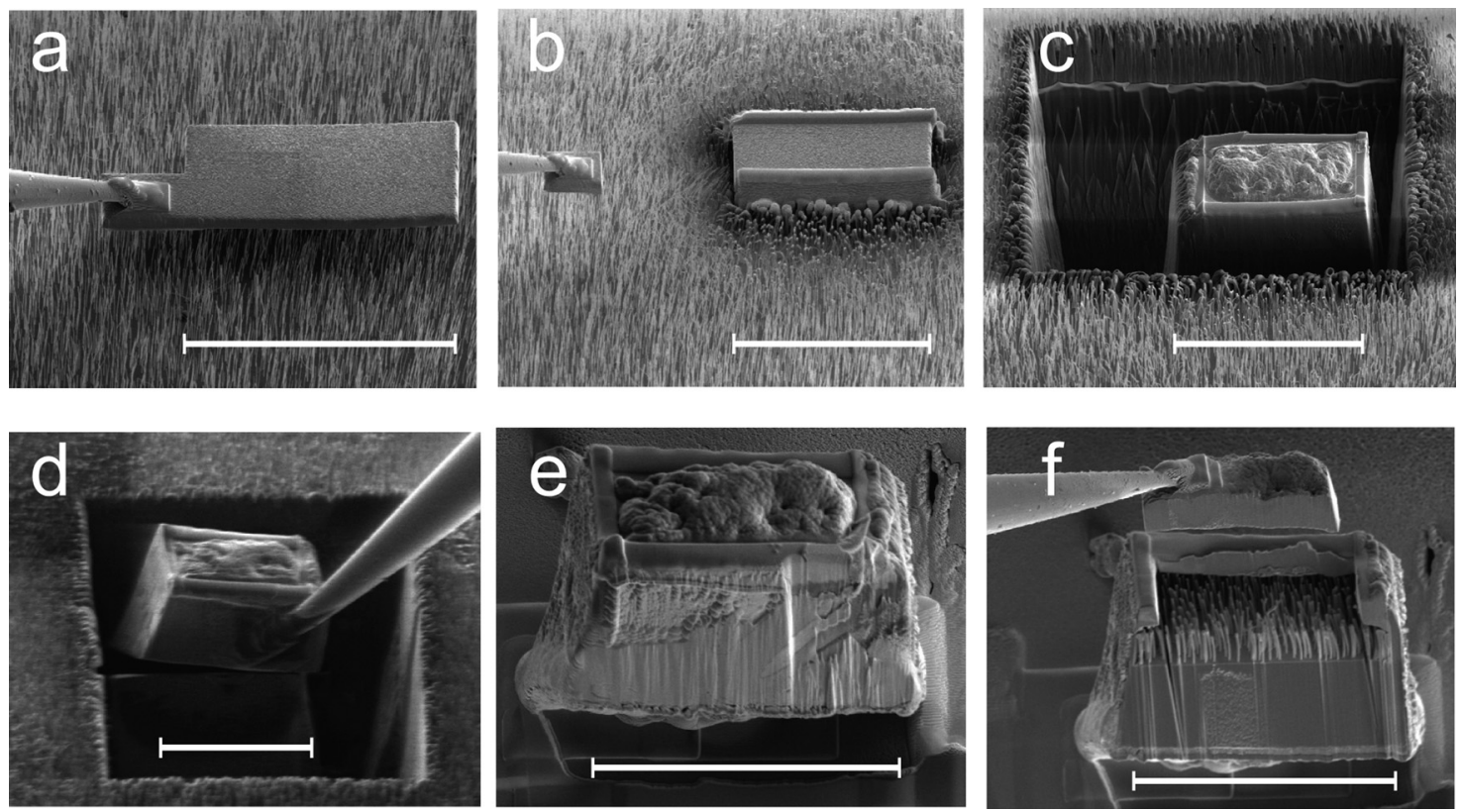

FIG. 2. Transfer of free-standing GaP nanowires. (a) Free-standing nanowires, with a $\sim 1 \mu$ m thick Au-shield positioned on top of the nanowires using a micromanipulator. (b) Au-shield attached via W-deposition on top of the nanowires, and the micromanipulator detached from the shield. (c) The nanowires under the Au-shield were sealed via additional W-deposition in order to avoid any damage during the following processing; a block containing these sealed nanowires was cut out of the substrate. (d) The release from the substrate of the block encapsulating the nanowires, with the micromanipulator attached to the block. (e) Block with nanowires attached to a new locus. (f) Nanowires revealed after the manipulation by removing the shield. SEM images: (a)-(c), (e), and (f) and FIB image: (d). Scale bar is $20 \mu \mathrm{m}$.

special block for further manipulations. The micromanipulator was attached to this block, which was then released from the substrate via FIB milling [Fig. 2(d)], transferred to a new locus with the micromanipulator, and attached there using W-FIB-CVD [Fig. 2(e)]. After cutting one side of the block and removing the Au-shield via FIB and the micromanipulator, the free-standing NWs were revealed and examined under SEM [Fig. 2(f)].

\section{B. Characterization of test device electrical contacts}

In order to evaluate electrical contacts which may be established to components assembled using the presented method, we also developed test devices made of Au film.

Gold test devices were cut from $\sim 1 \mu \mathrm{m}$ thick Au film loosely sitting on substrate. Details of this Au film formation are outlined above in Sec. II A. Figure 3(a) shows a test device cut out of and separated from the Au film. These test devices also contained a special bridge with which to attach a micromanipulator via W-FIB-CVD, as well as a rectangular hole made using FIB milling. This hole enabled four point resistance measurements to be made for electrical contacts to the test devices.

The test devices were transferred with the micromanipulator and attached via W-FIB-CVD to a new locus equipped with a set of metal electrodes for the four point contact resistance measurements [Fig. 3(b)]. ${ }^{36}$ The sets of four $\mathrm{Au}$ electrodes for these measurements were constructed on a bulk piece of $\mathrm{GaP}$ substrate $\left(15 \times 15 \mathrm{~mm}^{2}, 300 \mu \mathrm{m}\right.$ thick $)$ covered with $50 \mathrm{~nm}$ of hafnium oxide $\left(\mathrm{HfO}_{2}\right)$. The oxide layer was grown using the atomic layer deposition technique in a Savannah-100 system from Cambridge NanoTech in order to isolate the Au electrodes from the GaP substrate. The Au electrodes were composed of $15 \mathrm{~nm}$ titanium (Ti) and $75 \mathrm{~nm}$ Au film deposited on top of the $\mathrm{HfO}_{2}$ layer via magnetron sputtering (Orion 5, AJA International). Electrode shape was defined using ultraviolet lithography (300s exposure, Mask aligner MJB 4 DUV, Karl Süss MicroTec AG) on a $2.7 \mu \mathrm{m}$ thick layer of positive photosensitive Microposit S-1818 polymer (Shipley Company) spincoated and soft baked on a hot plate at $115^{\circ} \mathrm{C}$ for $90 \mathrm{~s}$. The resist development was done for $90 \mathrm{~s}$ in Microposit MF 319 (Shipley Company) developer. Then, the excess metal was etched through the polymer mask with Au etch $(10 \mathrm{~g} \mathrm{KI}$, $\left.2.5 \mathrm{~g} \mathrm{I}_{2}, 100 \mathrm{ml} \mathrm{H}_{2} \mathrm{O}, 30 \mathrm{~s}\right)$ and Ti etch (5\% v/v HF water solution, $10 \mathrm{~s}$ ), followed by resist removal with Microposit Remover 1165 at $80^{\circ} \mathrm{C}$ for $15 \mathrm{~min}$. Samples were cleaned in ozone for $2 \mathrm{~h}$ in order to ensure that the electrode surface was free of organic contamination.

The test devices were positioned over and mechanically pressed toward the electrodes, before being attached to the substrate via W-FIB-CVD. After being disconnected from the test devices using FIB milling, the micromanipulator $\mathrm{W}$-needle was employed to press different parts of the test device toward the substrate over different electrodes, in order to enable the formation of good mechanical contacts with the electrodes. These mechanical contacts were maintained using W-FIB-CVD. Finally, two FIB cuts were made in order to divide the test device into two separate pieces for the four point probe contact resistance measurements. Figure 3(b) shows a test device transferred to a set of Au electrodes, together with the schematic electrical circuit used for the four point contact resistance measurements. This figure also 

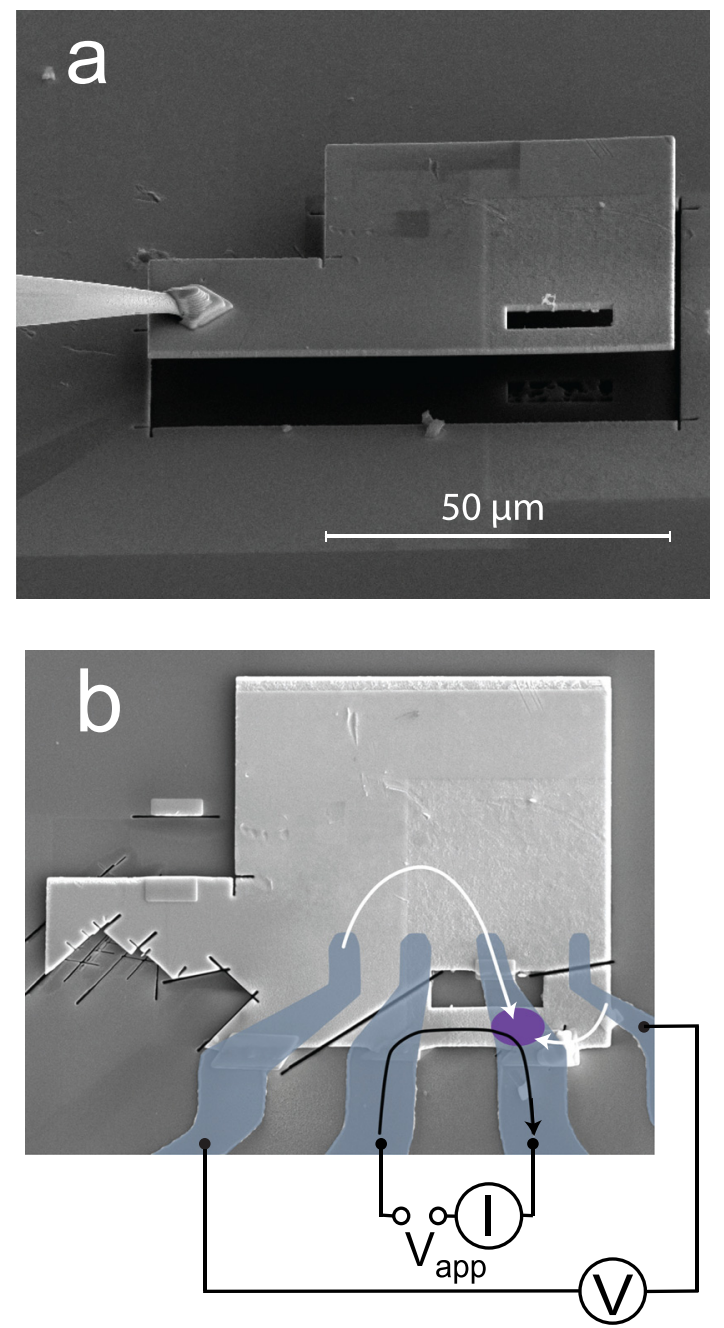

FIG. 3. (Color online) Four-point probe contact resistance measurements for a test device. (a) Scanning electron microscope image of the test device cut out of and separated from $1 \mu \mathrm{m}$ thick Au film, 52 tilt. (b) Top view of the same test device transferred using a micromanipulator to a set of Au electrodes, and a schematic of the electrical circuit used for the measurements. Black arrow: current flow; white arrows: voltage probing; circular area: contact area for which the contact resistance was measured; semitransparent shapes: locations of the Au electrodes below the test device. The scale is the same for both panels.

depicts the contact area for which the resistance was measured (the circular area), as well as the current flow (black arrow) and voltage probes (white arrows).

The contact resistance measured via the four point probe technique across a $\sim 30 \mu \mathrm{m}^{2}$ contact area was found to equal $<0.1 \Omega$, which corresponds to a specific contact resistance of $<10^{-8} \Omega \cdot \mathrm{cm}^{2}$. For comparison, NW-based electrodes employed to make recordings in the brain have $\sim 1 \mathrm{M} \Omega \mathrm{im}$ pedance at $1 \mathrm{kHz},{ }^{17}$ while a specific contact resistance of $<10^{-6} \Omega \cdot \mathrm{cm}^{2}$ is considered to be good for ohmic contacts to electronic devices. ${ }^{37}$ The tested contacts exhibited stable ohmic behavior, again indicating good contact quality.

\section{DISCUSSION AND CONCLUSIONS}

The experimental results demonstrate that the approach suggested for the direct 3D functional assembly of individual nanostructure-based components is feasible and one way to achieve it is the encapsulation of individual nanostructurebased components into special blocks. The following key features for the blocks can be identified: (1) protection of the component during the assembly process; (2) a means for the micro-/nanomanipulator attachment; (3) a means for achieving functional interconnection, e.g., electrical contacts; (4) a means enabling the component to exhibit its functionality after the assembly process. The key features (1) and (4) were demonstrated with the free-standing NWs, while the key features (2) and (3) were demonstrated using test devices made of Au film.

This demonstration of conceptual feasibility of all principal steps for a functional 3D assembly process opens the way for assembling minimally invasive neuronal probes for in vivo applications, as, e.g., outlined in Fig. 1. Potentially, this approach may also enable assembly of nanostructurebased components into other complex devices. In a further study, we will investigate different approaches of each individual step in order to develop a set of procedures tailored to various specific applications.

The key features (1), (3), and (4) appear to be necessary and sufficient for the assembly process to enable functional system integration. The feature (2) may provide clear benefits for the process, as shown with the test devices and at the same time can be potentially left out in some cases.

In the present study, the assembly process was performed via FIB-SEM. Obviously, due to the high costs associated with this technique, this is not an economically feasible approach. However, the disclosed assembly process can be robotized as well as potentially be carried out using low-cost optical microscopy in a protective atmosphere with, e.g., optical tweezers ${ }^{38}$ or micro- and nanomanipulators. ${ }^{39}$ And even more resource efficient parallel and unsupervised assembly processes can be also potentially adopted for this. ${ }^{26-29}$ Hence, the assembly process may be further streamlined substantially lowering the associated costs.

The suggested approach offers high added value particularly for applications with tough size constraints, such as complex micro- and nano-electromechanical systems ${ }^{40,41}$ and implantable devices. ${ }^{12,17}$ Smart (bionic) contact lenses-contact lenses with added electronic functionality for, e.g., continuous glucose level monitoring ${ }^{42-46}$-may also benefit from this fabrication approach, since bionic contact lenses need to preserve their flexibility, softness, transparency, strength against mechanical damage, provide liquid and oxygen penetration, and at the same time integrate heterogeneous elements to form a complete and complex functional system at an overall thickness of below 100-200 $\mu \mathrm{m}$. We envision that our approach may enable the development of a number of unique devices which may otherwise be unattainable. Application of this assembly process to the fabrication of biomedical devices such as implantable neural interfaces may justify its economic applicability for practical applications. However, applying our approach using more economical tools, e.g., under an optical microscope in air, and demonstrating the functionality of the assembled devices is also crucial for further development. In the long run, it 
will be important to identify the key features necessary or sufficient for a more general process of heterogeneous integration. This can be achieved after the process demonstration with different tools using different nanostructure-based components. We expect that the key features presented in this report will be generic for this assembly process.

To summarize, we have suggested and tested a concept and an approach aimed at the direct 3D functional assembly of individual nanostructure-based components into brain implants. We have proved the feasibility of this assembly by transferring specially designed blocks containing freestanding NWs to another substrate. We have also shown that the contact resistance for components assembled in this way is suitable for device applications. We have discussed the key features essential for successful functional assembly. Our approach holds promise that minimally invasive neural probes operating in vivo for extended periods of time (chronic probes) can be fabricated as suggested. Even if one generally would need to further develop the method for economic of scale reasons, in our particular case of neural probes fabrication, a high cost can be easily justified balanced the need for minimal size requirements. Further development of this approach may enable assembly of a wider range of complex devices composed of heterogeneous nanostructures, in this way opening up a new avenue toward the fabrication of systems of nanosystems.

\section{ACKNOWLEDGMENTS}

This work was supported by the Swedish Research Council (621-2009-3266, 2013-6006, and Linnaeus Grant No. 80658701), the Knut and Alice Wallenberg Foundation (KAW 2004.0119), and the Nanometer Structure Consortium at Lund University (nmC@LU).

${ }^{1}$ A. A. Requicha, Proc. IEEE 91, 1922 (2003).

${ }^{2}$ B. Gyselinckx, C. Van Hoof, J. Ryckaert, R. F. Yazicioglu, P. Fiorini, and V. Leonov, Proceedings of the IEEE 2005: Custom Integrated Circuits Conference, San Jose, CA, September 18-21, 2005 (IEEE, Piscataway, NJ, 2005), pp. 13-19.

${ }^{3}$ C. McCaffrey, O. Chevalerias, C. O'Mathuna, and K. Twomey, Pervasive Computing, IEEE 7, 23 (2008).

${ }^{4}$ H. Ho, E. Saeedi, S. S. Kim, T. Shen, and B. A. Parviz, Proceedings of the IEEE Micro Electro Mechanical System, Tucson, AZ, January 13-17, 2008 (IEEE, Piscataway, NJ, 2008), p. 403.

${ }^{5} \mathrm{G}$. Troster, Pervasive Computing, IEEE 10, 87 (2011).

${ }^{6}$ J. A. Rogers, JAMA-J. Am. Med. Assoc. 313, 561 (2015).

${ }^{7}$ B. Warneke, M. Last, B. Liebowitz, and K. S. Pister, Computer 34, 44 (2001).

${ }^{8}$ L. Smith, Chip Scale Rev. 12, 51 (2008).

${ }^{9}$ D. J. Barnhart, T. Vladimirova, and M. N. Sweeting, J. Spacecr. Rockets 44, 1294 (2007).

${ }^{10}$ R. Martinez-Val and E. Perez, Proc. Inst. Mech. Eng. Part C 223, 2767 (2009).

${ }^{11}$ B. Tian and C. M. Lieber, Annu. Rev. Anal. Chem. 6, 31 (2013).

${ }^{12}$ T. I. Kim et al., Science 340, 211 (2013).
${ }^{13}$ J. Schouenborg, Progress in Brain Research, edited by J. Schouenborg, M. Garwicz, and N. Danielsen (Elsevier B.V., Amsterdam, Netherlands, 2011), Vol. 194, pp. 61-70.

${ }^{14}$ N. A. Kotov et al., Adv. Mater. 21, 3970 (2009).

${ }^{15}$ T. Bryllert, L.-E. Wernersson, L. Froberg, and L. Samuelson, IEEE Electron Device Lett. 27, 323 (2006).

${ }^{16}$ C. P. T. Svensson, T. Mårtensson, J. Trägårdh, C. Larsson, M. Rask, D. Hessman, L. Samuelson, and J. Ohlsson, Nanotechnology 19, 305201 (2008).

${ }^{17}$ D. B. Suyatin, L. Wallman, J. Thelin, C. N. Prinz, H. Jörntell, L. Samuelson, L. Montelius, and J. Schouenborg, PLoS One 8, e56673 (2013).

${ }^{18}$ V. Andoralov et al., Sci. Rep. 3, 3270 (2013).

${ }^{19}$ S. A. Stauth and B. A. Parviz, Proc. Natl. Acad. Sci. U. S. A. 103, 13922 (2006).

${ }^{20}$ H. Keum, A. Carlson, H. Ning, A. Mihi, J. D. Eisenhaure, P. V. Braun, J. A. Rogers, and S. Kim, J. Micromech. Microeng. 22, 055018 (2012).

${ }^{21}$ M. Gullo, L. Jacot-Descombes, and J. Brugger, Proceedings of the 26th International IEEE MEMS Conference, Taipei, Taiwan, January 20-24, 2013 (IEEE, Piscataway, NJ, 2013), pp. 78-81.

${ }^{22}$ Y. Zhang, H. Keum, K. Park, R. Bashir, and S. Kim, J. Microelectromech. Syst. 23, 308 (2014).

${ }^{23}$ G. A. Salvatore, N. Münzenrieder, T. Kinkeldei, L. Petti, C. Zysset, I. Strebel, L. Büthe, and G. Tröster, Nat. Commun. 5, 2982 (2014).

${ }^{24}$ S. Xu et al., Science 344, 70 (2014).

${ }^{25}$ Y. L. Kong et al., Nano Lett. 14, 7017 (2014).

${ }^{26}$ M. Mastrangeli, S. Abbasi, C. Varel, C. Van Hoof, J.-P. Celis, and K. F. Böhringer, J. Micromech. Microeng. 19, 083001 (2009).

${ }^{27}$ Y. Ke, L. L. Ong, W. M. Shih, and P. Yin, Science 338, 1177 (2012).

${ }^{28}$ S. C. Park, J. Fang, S. Biswas, M. Mozafari, T. Stauden, and H. O. Jacobs, Adv. Mater. 26, 5942 (2014).

${ }^{29}$ M. Collet, S. Salomon, N. Y. Klein, F. Seichepine, C. Vieu, L. Nicu, and G. Larrieu, Adv. Mater. 27, 1268 (2015).

${ }^{30} \mathrm{G}$. Van der Plas et al., IEEE J. Solid-State Circuits 46, 293 (2011).

${ }^{31}$ W. Hällström, T. Mårtensson, C. Prinz, P. Gustavsson, L. Montelius, L. Samuelson, and M. Kanje, Nano Lett. 7, 2960 (2007).

${ }^{32}$ W. Hällström, C. N. Prinz, D. Suyatin, L. Samuelson, L. Montelius, and M. Kanje, Langmuir 25, 4343 (2009).

${ }^{33}$ A. A. Tseng, Small 1, 924 (2005).

${ }^{34}$ M. H. Magnusson, K. Deppert, J.-O. Malm, J.-O. Bovin, and L. Samuelson, J. Nanopart. Res. 1, 243 (1999).

${ }^{35}$ D. B. Suyatin, W. Hällström, L. Samuelson, L. Montelius, C. N. Prinz, and M. Kanje, J. Vac. Sci. Technol. B 27, 3092 (2009).

${ }^{36}$ D. K. Schroder, Semiconductor Material and Device Characterization, 3rd ed. (Wiley-Interscience, New York, 2006).

${ }^{37}$ T. Shen, G. Gao, and H. Morkoc, J. Vac. Sci. Technol. B 10, 2113 (1992).

${ }^{38}$ P. J. Pauzauskie, A. Radenovic, E. Trepagnier, H. Shroff, P. Yang, and J. Liphardt, Nat. Mater. 5, 97 (2006).

${ }^{39} \mathrm{~K}$. Mølhave, T. Wich, A. Kortschack, and P. Bøggild, Nanotechnology 17, 2434 (2006).

${ }^{40}$ A. Ludwig, J. Cao, J. Brugger, and I. Takeuchi, Meas. Sci. Technol. 16, 111 (2005).

${ }^{41}$ J.-W. Jeong, B. Park, H. Keum, S. Kim, J. A. Rogers, and O. Solgaard, Opt. Express 21, 13800 (2013).

${ }^{42}$ H. Yao, A. J. Shum, M. Cowan, I. Lähdesmäki, and B. A. Parviz, Biosens. Bioelectron. 26, 3290 (2011).

${ }^{43}$ M. Falk, V. Andoralov, Z. Blum, J. Sotres, D. B. Suyatin, T. Ruzgas, T. Arnebrant, and S. Shleev, Biosens. Bioelectron. 37, 38 (2012).

${ }^{44}$ N. Thomas, I. Lähdesmäki, and B. A. Parviz, Sens. Actuators B: Chem. 162, 128 (2012).

${ }^{45}$ H. Yao, Y. Liao, A. Lingley, A. Afanasiev, I. Lähdesmäki, B. Otis, and B. Parviz, J. Micromech. Microeng. 22, 075007 (2012).

${ }^{46}$ Y.-T. Liao, H. Yao, A. Lingley, B. Parviz, and B. P. Otis, IEEE J. SolidState Circuits 47, 335 (2012). 